\title{
Modelling Q-factors of micro pillars
}

Nielsen, Torben Roland; Gregersen, Niels; Tromborg, Bjarne; Mørk, Jesper

Published in:

9th International Conference on Transparent Optical Networks, 2007. ICTON '07.

Link to article, DOI:

10.1109/ICTON.2007.4296176

Publication date:

2007

Document Version

Publisher's PDF, also known as Version of record

Link back to DTU Orbit

Citation (APA):

Nielsen, T. R., Gregersen, N., Tromborg, B., \& Mørk, J. (2007). Modelling Q-factors of micro pillars. In 9th International Conference on Transparent Optical Networks, 2007. ICTON '07. IEEE.

https://doi.org/10.1109/ICTON.2007.4296176

\section{General rights}

Copyright and moral rights for the publications made accessible in the public portal are retained by the authors and/or other copyright owners and it is a condition of accessing publications that users recognise and abide by the legal requirements associated with these rights.

- Users may download and print one copy of any publication from the public portal for the purpose of private study or research.

- You may not further distribute the material or use it for any profit-making activity or commercial gain

- You may freely distribute the URL identifying the publication in the public portal

If you believe that this document breaches copyright please contact us providing details, and we will remove access to the work immediately and investigate your claim. 


\title{
Modelling Q-Factors of Micro Pillars
}

\author{
T. R. Nielsen, N. Gregersen, B. Tromborg and J. Mørk \\ COM $\cdot D T U$, Department of Communications, Optics \&Material, Nano $\cdot D T U$ \\ Technical University of Denmark, Building 343, DK-2800 Kgs. Lyngby, Denmark \\ Tel: (+45) 4525 6367, Fax: (+45) 4525 6367, e-mail: trn@com.dtu.dk
}

\begin{abstract}
The influence of fabrication induced imperfections on quality factors for a microcavity pillar is studied numerically. The dependence on side-wall inclination and etch variations is quantified.

Keywords: microstructure devices, resonators, photonic integrated circuits.
\end{abstract}

\section{INTRODUCTION}

Semiconductor micro-cavity pillars (MP's) based on distributed Bragg reflectors (DBR's) are expected to play an important role for the realization of single photon sources [1-4]. The emission properties of a semiconductor quantum dot placed inside the micro-cavity depends strongly on the optical properties of the micro-cavity and thus the rate of spontaneous emission rate can be enhanced or suppressed compared to the decay rate in free space. This is quantified by the Purcell-factor which is proportional to the ratio of the quality (Q) factor over the effective mode volume. The Q-factor depends critically on the precise geometry and material composition of the micropost investigated. In practice, the Q-factor will be hampered by different kinds of fabrication induced imperfections as well as inherent absorption in the structures. For ideal MP's, see Fig. 1., with uniform radius along the growth direction the Q-factor variation with pillar diameter and cavity length has previously been studied [5,6]. Only recently the role of imperfect structures has been addressed [7,8]. We extend these studies by investigating the influence of the sidewall inclination and non-uniformity. Two types of geometries (Fig. 2) mimicking fabrication-induced imperfections are investigated. For type I, the MP has a conical shape corresponding to the effect of underetch from reactive ion etching. For type II, the AlAs layers have a smaller diameter than the GaAs layers, simulating the selective oxidation-assisted underetch of the AlAs layers from the sidewalls reported in Ref. [9].

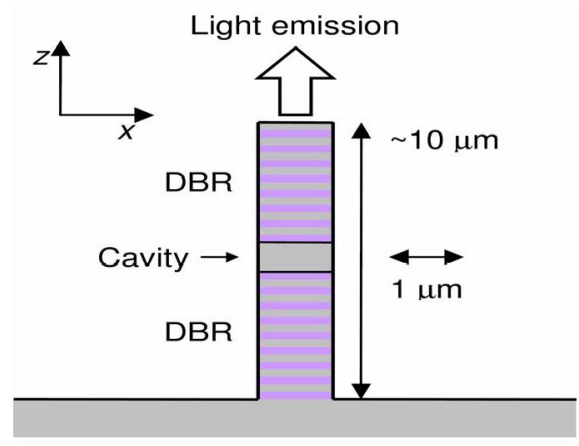

Figure 1. (Color online) Sketch of ideal micro pillar.
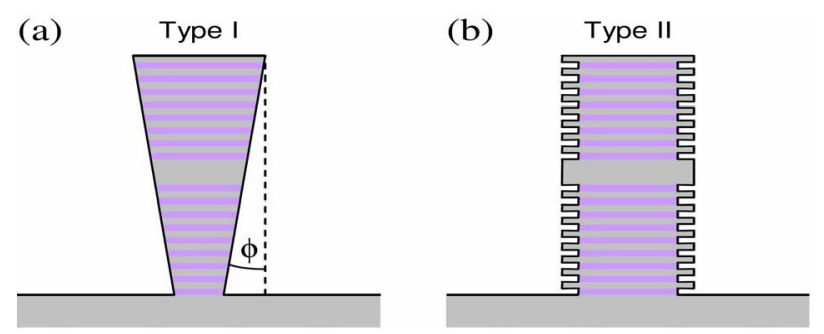

Figure 2. (Color online) Illustrations of the two pillar geometry imperfections considered.

\section{THEORY}

We use the eigenmode expansion technique [10] (EET) to study the Q-factors. The EET allows a full threedimensional vectorial solution of the wave equation, necessary when considering MP's of small diameters and in general whenever diffraction effects are dominating. For this frequency domain technique, the MP structure is divided into layers of uniform refractive index profile along a propagation axis, usually chosen as the $z$ axis. In each layer, eigenmodes are determined for the particular index profile assuming uniformity along the entire $z$ axis. In cylindrical coordinates $(r, \theta, z)$, an eigenmode with propagation constant $\beta$ and angular momentum $l$ is of the general form $\mathbf{E}(\mathbf{r})=\mathbf{e}(r) \exp (i l \theta+i \beta z)$. The optical field in each layer is expanded on the corresponding eigenmodes, and the fields at each side of the interface between adjacent layers are connected using the scattering matrix formalism.

In general, two incoming fields with expansion coefficients $\boldsymbol{u}^{(0)}$ and $\boldsymbol{d}^{(p+1)}$ separated by a structure of $p$ layers are connected by the scattering matrix $S^{(p)}$ to the outgoing fields $\boldsymbol{u}^{(p+1)}$ and $\boldsymbol{d}^{(0)}$. The S-matrix describes the total reflection and transmission through the structure with $p$ layers as [11] 


$$
\left[\begin{array}{c}
\mathbf{u}^{(p+1)} \\
\mathbf{d}^{(0)}
\end{array}\right]=\left[\begin{array}{ll}
T_{u u}^{(p)} & R_{u d}^{(p)} \\
R_{d u}^{(p)} & T_{d d}^{(p)}
\end{array}\right]\left[\begin{array}{c}
\mathbf{u}^{(0)} \\
\mathbf{d}^{(p+1)}
\end{array}\right]=S^{(p)}\left[\begin{array}{c}
\mathbf{u}^{(0)} \\
\mathbf{d}^{(p+1)}
\end{array}\right] .
$$

The Q factor is calculated in the following way. Light propagating through the cavity is partially reflected at the top and bottom DBR mirrors and the sum of this infinite series of reflections is given by the total roundtrip operator

$$
M=\left(1-R^{t} \Theta R^{b} \Theta\right)^{-1},
$$

where $R^{t, b}$ are matrices describing reflection at the top and bottom DBR mirror respectively, while $\Theta$ is the translation operator propagating the modes from one end of the cavity to the other. The transmission operator of the entire MP is defined by

$$
T=T^{b} \Theta M T^{t},
$$

with $T^{t, b}$ describing transmission through the top and bottom DBRs, respectively. Since the quantities $T^{t, b}$ and $\Theta$ vary slowly compared to the roundtrip operator $M$, a resonance in $M$ corresponds to a resonance in the transmitted light spectrum. The inverse of the quantity $\left|\operatorname{det}\left(M^{-1}\right)\right|^{2}$ is then calculated as function of wavelength, a resonance peak is identified and the center position and the FWHM width are extracted to obtain the Q factor.

\section{RESULTS}

We consider a GaAs/AlAs MP with 26 and 291/2 layer pairs in the top and bottom DBR-mirror using typical wavelength-independent refractive indices of 3.542 (2.958) for GaAs (A1As). The pillar diameter for the ideal geometry is $1 \mu \mathrm{m}$ with GaAs (AlAs) layer thickness of 72 (79) $\mathrm{nm}$ and a cavity thickness of $294 \mathrm{~nm}$. The structure, which is similar to those considered in Refs. $[4,12]$, has a primary resonance wavelength of $\sim 969 \mathrm{~nm}$ and a Q-factor of $\sim 12,000$. In what follows we compare this standard cavity design, where the layer thickness is typically chosen on the basis of a one-dimensional planar-cavity transfer matrix calculation, to an optimized design where the layer thicknesses are determined by calculating the effective index $n_{\text {eff }}$ of the fundamental $\mathrm{HE}_{11}$ mode and by setting the layers thicknesses equal to $\lambda /\left(4 n_{\text {eff }}\right)$ and the cavity thickness equal to $\lambda / n_{\text {eff. In our }}$ simulations, the optimization design wavelength $\lambda$ was $950 \mathrm{~nm}$.

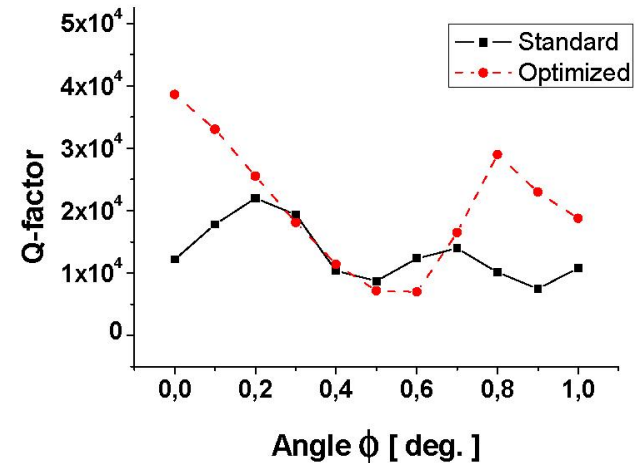

Figure 3. (Color online) Type I imperfection MP. The $Q$-factor for standard and optimized structure.
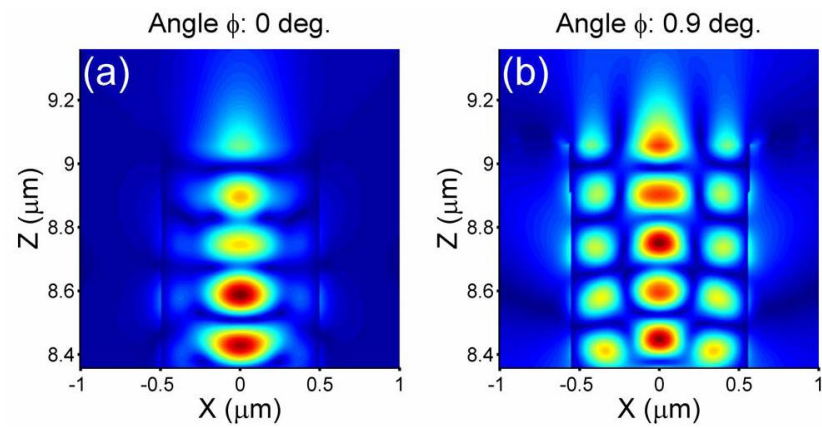

Figure 4. (Color online) Radial electric field profiles in top $D B R$ mirror for an inclination angle $\phi$ of $0^{\circ}(a)$ and $0.9^{\circ}(b)$ for the type I optimized MP structure.

First we consider the conical shaped MPs [13] and plot the Q-factor as a function of the inclination angle in Fig. 3. The results for the standard design are shown in the solid line, while the optimized design is shown in the dotted line. In both cases the Q-factor varies non-monotonously with increasing angle of inclination. For the standard design, the Q-factor increases for small angles and reaches a maximum value at a non-zero degree $\left(\phi=0.2^{\circ}\right)$, followed by a minor peak at larger angles $\left(\phi=0.7^{\circ}\right)$. These increases in $\mathrm{Q}$ are somewhat counterintuitive as one would expect imperfections to have a negative influence on the Q-factors. Furthermore, the Q-factor changes in magnitude by almost a factor of 3 within a variation in angle of just one deg. For the optimized structure the peak at $0.2^{\circ}$ has disappeared, while the second minor peak, now shifted to $0.8^{\circ}$, is still present. Thus, for the standard design the small angle increase in the Q-factor can be attributed to the fact that the inclination angle producing a perturbation of the DBR mirror reflectivity towards its optimum value.

To identify the origin of the second peaks we examine the field profiles of the MP for the optimized design. The field profile for the ideal structure $(\phi=0)$ depicted in Fig. $4 a$ shows a fundamental mode propagating through the top DBR layers, while the field profile for the MP with an large inclination angle shown in Fig. $4 b$ 
displays coupling to higher or der modes. Qualitatively, for non-zero inclination angle the interaction between the $\mathrm{HE}_{11}$ mode and higher order modes can assist in maintaining a high Bragg mirror reflectivity. This is in accordance with previous studies $[5,6]$, where a similar higher order mode interaction, responsible for strong variations in the $\mathrm{Q}$-factor, was also identified. Finally, we note that the relative change in the $\mathrm{Q}$-factor is larger for the optimized structure than for the standardMP design

F or the underetched MPs the Q-factor as a function of the etch depth is shown in Fig 5 for both the normal and the optimized MP design. The peak near an etch of $10 \mathrm{~nm}$ is only shifted slightly in the optimized structure (dashed line) as illustrated in Fig 5, and the iritial increase of Q with A1As underetch is still present as for the unoptimized structure (solid line). These increases in $\mathrm{Q}$ are somewhat counter-intuitive as one would expect imperfections to have a negative influence on the $\mathrm{Q}$ factor. To study the origin of this increase, we have in addition evaluated the $\mathrm{Q}$ factor as function of an artificial under etches into the GaAs layers (dotted line). This type of underetch is not observed experimentally, but we introduce it to study the physical mechanism behind the increase in $\mathrm{Q}$. For the $\mathrm{G}$ aAs underetch, we observe a monotoric reduction in $\mathrm{Q}$ in Fig. 5 with increasing etch and no peak is present. This behavior can be understood by considering the influence of the etch depth on the

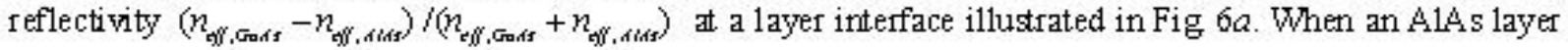
diameter is reduced the mode is pushed out of the pillar and the effective index drops towards unity. The effective index contrast between the GaAs and A1As layers becomes larger and the DBR reflectivity is thus improved resulting in an increase in $\mathrm{Q}$. Similarly, the effective index of the GaAs layer becomes smaller when the diameter is decreased however, in this case the index contrast is reduced and the DBR mirror reflectivity is only lowered. In this way, underetching of the A1As (GaAs) 1ayers causes an improvement (recuction) in Q. H owever, in an analysis of the DBR reflectivity, the overlap integral of the transverse components of the $\mathrm{EH}_{11}$ modes in $\mathrm{G}$ aAs and $\mathrm{A} 1 \mathrm{~A}$ s layers, given by

$$
O=\left|\int \mathbf{E}_{\perp, G a d s} \cdot \mathbf{E}_{\perp, \text { i iss }} d \mathbf{r}_{\perp}\right|
$$

should also be considered. Fig. $6 b$ illustrates the overlap as function of etch into the GaAs or A1As layers. In both cases the mode overlap deterior ates with decreasing layer diameter, at one point the effect of the poor overlap dominates over the effect of increased reflectivity for AlAs underetch and the $Q$ then drops from its peak value. We note that the value of $\mathrm{Q}$ at its peak for the type II imperfection is $\sim 25 \%$ higher than that of the ideal structure. Experimental control of the A1As sidewall underetch would thus allow an increase in $Q$ and would be beneficial for realizing high-Q MPs.

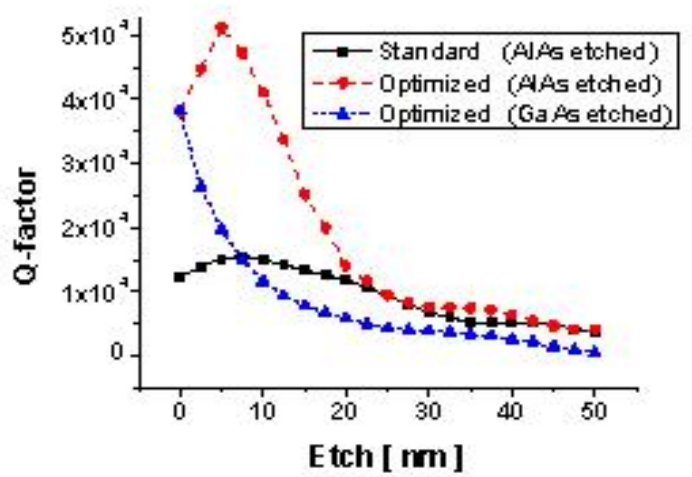

Figure 5. (Color onine) Type II imperfection MP. Q- factor for standar d and optimized MP structire
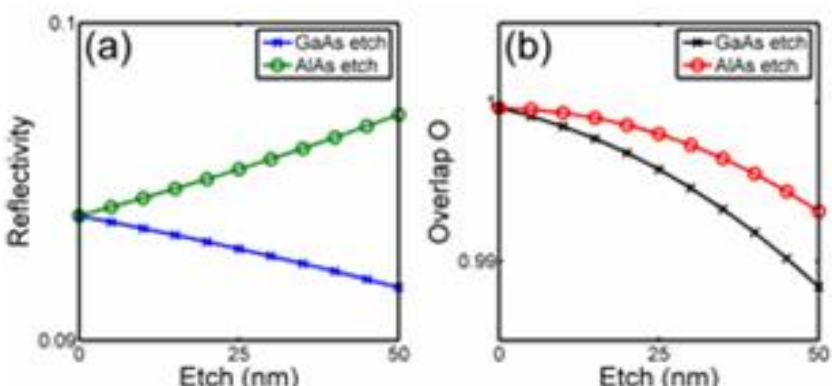

Figure 6 . (Color onine) Feflectivity (a) and overiap of $H E_{11}$ modes in GaAs and AiAs layers

(b) as function of etch depth

\section{CONCLUSIONS}

In summary, we have studied the influence of fabrication-induced imperfections on the $Q$-factor of MPs and our results show that the variations in $Q$ can be attributed to a delicate balance between effective index contrasts, mode-overlap and higher-order mode contributions. Fabrication tolerance recuirements for MP Q-factors can be estimated on the basis of our findings.

\section{ACKNOWLEDGEMENTS}

The work was supported by the EU IST project "QPhoton" (Contract no.: IST-29283) and we thank A. Löfler andS. Reitzenstein and for fruitful discussions. The authors also acknowledge access to the Sun HPC systems at 
the Technical University of Denmark, made possible by a collaboration with the Scientific Computing Group at IMM•DTU under the grant number DCSC HDW-1104-08.

\section{REFERENCES}

[1] I. Robert, E. Moreau, J. M. Gérard, and I Abram, "Towards a single-mode photon source based in single quantum dots", J. Lum. 94-95, 797, (2001).

[2] J.M. Gérard, B. Gayral, and E. Moreau, "On the efficacy of solid-state single photon sources", quant$\mathrm{ph} / 0207115$.

[3] K. J. Vahala, "Optical Microcavities", Nature 424, 839 (2003).

[4] J. P. Reithmaier, et al., "Strong coupling in a single quantum dot-semiconductor microcavity system", Nature, 432, 197 (2004)

[5] P. Lalanne, J. P. Hugonin and J.M. Gérard, "Electromagnetic study of the quality factor of pillar microcavities in the small diameter limit", App. Phys. Lett. 84, 4726 (2004).

[6] G. Lecamp, P. Lalanne, J. P. Hugonin and J.-M. Gérard, "Energy transfer through laterally confined Bragg mirrors and its impact on pillar microcavities", IEEE J. Quantum Electron. 41, 1323 (2005).

[7] J. Vučković, M. Pelton, A. Scherer and Y. Yamamoto, "Optimization of three-dimensional micropost microcavity for cavity quantum electrodynamics", Phys. Rev. B 66, 023808 (2002).

[8] G. Lecamp, J.P. Hugonin and P. Lalanne, "Submicron-diameter semiconductor pillar microcavities with very high quality factors", Appl. Phys. Lett. 90, 091120 (2007).

[9] S. Varoustis, et al., "Reactive etching of high-Q and submicron-diameter GaAs/AlAs micropillar cavities", J. Vac. Sci. Technol. B, 26, 2499 (2005).

[10] P. Bienstman, H. Derudder, R. Baets, F. Olyslager, D.D. Zutter, "Analysis of cylindrical waveguide discontinuities using vectorial eigenmodes and perfectly matched layers", IEEE Trans. Microw. Theory Tech. 49, 349-354 (2001).

[11] L. Li, "Formulation and comparison of two recursive matrix algorithms for modeling layered diffraction gratings", J. Opt. Soc. Am. A 13, 1024 (1996).

[12] A. Löffler et al., "Semiconductor quantum dot microcavity pillars with high-quality factors and enlarged dot dimensions", Appl. Phys. Lett. 86, 111105 (2005)

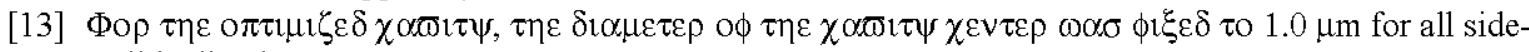
wall inclinations. 